\title{
Dy-Sb (Dysprosium-Antimony)
}

\section{H. Okamoto}

The Dy-Sb phase diagram in [Massalski2] was redrawn from [1980Mir]. [1988Fer] discussed that $\mathrm{DySb}_{2}$ may exist in a metastable state or under high pressures in view of other rare earth-antimony systems. [2010Lon] assessed this system thermodynamically. The data of [1980Mir] were used as the phase boundary data. $\alpha \mathrm{Dy}_{4} \mathrm{Sb}_{3}$ was stable down to low temperatures in [1980Mir] diagram. However, [2003Mor] found that this phase becomes unstable at low temperatures (already unstable at $827^{\circ} \mathrm{C}$ ). This feature is reflected in the Dy-Sb phase diagram calculated by [2010Lon] (Fig. 1).

The $\alpha$ DySb liquidus in Fig. 1 has an unusual shape on the $\mathrm{Sb}$ rich end. The liquidus would cross the $100 \% \mathrm{Sb}$ line when extrapolated to the $\mathrm{Sb}$-rich side unless an extreme change of slope is introduced. The occurrence of this type of situation is thermodynamically very unlikely [1991Oka]. The original phase diagram of [1980Mir] is preferred for the liquidus shape in this range. Further experimental data are required for confirmation. Some phase boundary data are available in addition to those used by [2010Lon], but they are not useful in settling this problem: [1967Gam] reported a Dy-Sb phase diagram, but it was for the Dy-rich side only. The phase diagram reported by [1979Mir] was apparently superseded by [1980Mir].

\section{References}

1967Gam: R.J. Gambino, Rare-Earth-Sb and -Bi Compounds with the $\mathrm{Gd}_{4} \mathrm{Bi}_{3}$ (anti- $\mathrm{Th}_{3} \mathrm{P}_{4}$ ) Structure, J. Less-Common Met., 1967, 12, p 344-352

1979Mir: K.E. Mironov, O.R. Burnashev, Phase Diagram of the Dy-Sb System, Dokl. Akad. Nauk SSSR, 1979, 245(5), p 11631166, in Russian; TR: Dokl. Phys. Chem., 1979, 245, p 333-335

1980Mir: K.E. Mironov, M.N. Abdusalyamova, O.R. Burnashev, The Phase Diagram of the Dy-Sb System, Izv. Akad. Nauk SSSR, Neorg. Mater, 1980, 16(11), p 1951-1956, in Russian; TR: Inorg. Mater, 1980, 16(11), p 1332-1336

1988Fer: R. Ferro, G. Borzone, G. Cacciamani, On the Thermochemistry of the Rare Earth Antimonides. The Dy-Sb System, Thermochim. Acta, 1988, 129, p 99-113

1991Oka: H. Okamoto, T.B. Massalski, Thermodynamically Improbable Phase Diagrams, J. Phase Equilib., 1991, 12(2), p 148-168

2003Mor: A.V. Morozkin, Dy-Sb-Si System at $1100 \mathrm{~K}$ and Ternary Intermetallic Phases in the Dy-Sb-Si and Gd-Sb-Si Systems, J. Alloys Compd., 2003, 358, p L6-L8

2010Lon: Z.H. Long, S.H. Zhou, H.S. Liu, Z.P. Jin, Thermodynamic Assessment of Ag-Dy-Sb Ternary System, Thermochim. Acta, 2010, 498, p 22-26

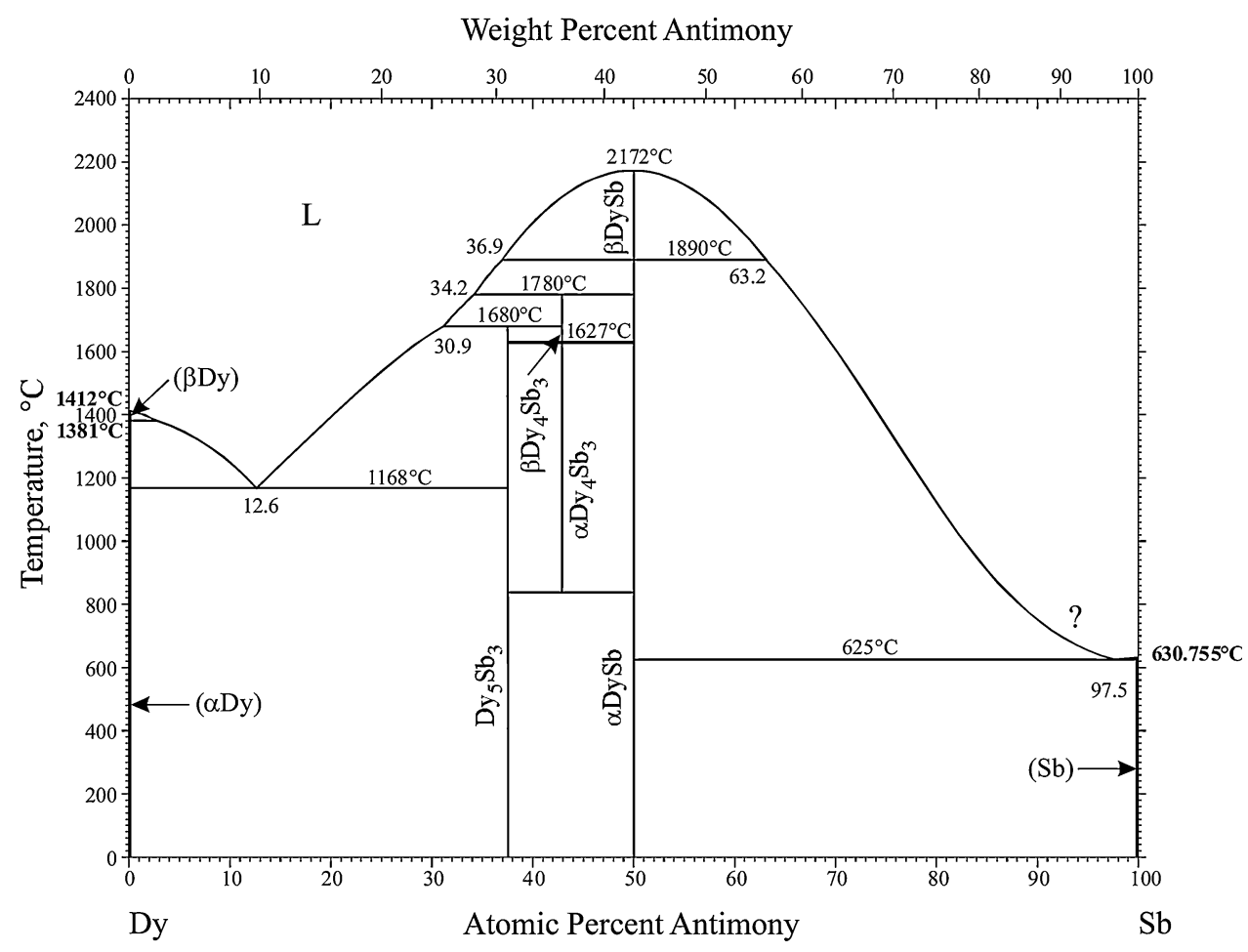

Fig. 1 Dy-Sb phase diagram 